\title{
Inflammation, atherosclerosis, and arterial thrombosis: Role of the scavenger receptor CD36
}

\section{ABSTRACT}

The CD36 scavenger receptor recognizes oxidized low-density lipoprotein (LDL) and cell-derived microparticles. It is expressed on macrophages and platelets and is a mediator of both atherogenesis and thrombosis. Macrophages from CD36-null mice have a defect in foam cell formation in response to exposure to oxidized LDL, and CD36-null mice fed an atherogenic Western diet have significantly less atherosclerosis than their wild-type counterparts. On platelets, CD36 recognition of oxidized LDL contributes to their activation and provides a mechanistic link between hyperlipidemia, oxidant stress, and the prothrombotic state. Cell-derived microparticles are also major ligands for CD36 and contribute to thrombus formation in a CD36-dependent manner even in the absence of hyperlipidemia. CD36 deficiency in mice is associated with inhibition of thrombus formation and with a reduction in microparticle accumulation in thrombi. Targeting CD36 is a promising avenue for the treatment of atheroinflammatory disorders.

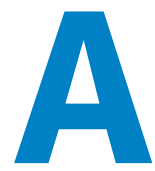
therosclerosis is recognized as a chronic inflammatory disorder of the vessel wall. Four categories of evidence support the model of atherosclerosis as an inflammatory disease:

- Biomarkers of inflammation are clearly associated with risk and prognosis of atherosclerosis. Three that have been linked conclusively are: C-reactive protein, myeloperoxidase (a marker of leukocyte activation), and antibodies to oxidative modifications of low-density lipoprotein (LDL).

Dr. Silverstein reported that he has no financial interests or relationships that pose a potential conflict of interest with this article.

This article was developed from an audio transcript of Dr. Silverstein's presentation at the 3rd Heart-Brain Summit. The transcript was formatted and edited by the Cleveland Clinic Journal of Medicine staff for clarity and conciseness, and was then reviewed, revised, and approved by Dr. Silverstein.

doi:10.3949/ccjm.76.s2.06
- Tissue studies demonstrate that leukocytes and products of the inflammatory system are prevalent in atherosclerotic plaque.

- Animal models show an absence of atherosclerosis in the absence of monocytes or monocyte recruitment as well as a crucial role for T-cell-derived proinflammatory cytokines.

- It is becoming apparent that patients with chronic systemic inflammatory disorders (eg, systemic lupus erythematosus, Wegener granulomatosis, chronic obesity, and aging) have increased risk of atherosclerosis.

This article examines the mechanisms by which inflammation promotes the development of atherosclerosis and coronary artery disease, with particular attention to the role of CD36, a scavenger receptor for oxidized LDL.

\section{OXIDATION IN PLAQUE FORMATION}

Prevailing models that link inflammation to plaque formation suggest that inflammatory stimuli (eg, cigarette smoke, hypertension) provoke changes in the phenotype of the cells of the arterial vessel wall that allow penetration of leukocytes and LDL particles across the endothelial barrier, trapping them in the subendothelial space. ${ }^{1,2}$ An inflammatory reaction then occurs in the subendothelial space involving monocytes/macrophages and lymphocytes (especially $\mathrm{T}$ cells). Ultimately, through the production and release of oxidizing enzymes such as myeloperoxidase and nitric oxide synthase, the reaction leads to generation of reactive oxygen and nitrogen species. In this setting, LDL particles become modified to a form known as oxidized LDL (oxLDL). OxLDL loses its ability to bind to LDL receptors, which interferes with its normal processing; perhaps more important, oxLDL gains an affinity for a family of proteins called scavenger receptors. Scavenger receptors on macrophages bind and internalize the oxLDL particles, leading to accumulation of cholesterol and other lipids in the cells. Over prolonged periods, increasing 
quantities of oxLDL become internalized, leading to formation of foam cells (lipid-laden macrophages), the precursor to atherosclerotic plaque. These lipidladen cells are more prone to apoptosis, which further contributes to plaque growth and rupture.

\section{CD36: A CRITICAL SCAVENGER RECEPTOR}

One of the most critical scavenger receptors on macrophages is CD36, which is a transmembrane glycoprotein that crosses the membrane twice. CD36 is expressed heavily on monocytes, macrophages, dendritic cells, fat, muscle, capillary endothelial cells, and platelets. It has multiple physiologic functions, including acting as a high-affinity receptor for specific oxidized phospholipids that are found within oxLDL. ${ }^{3}$ It is also a receptor for phosphatidyl serine (PS) and oxidized PS (oxPS) that is expressed on the surface of apoptotic cells. CD36 is highly conserved in evolution; orthologs are even found in flies, worms, and sponges. Evidence suggests that CD36 and other scavenger receptors probably evolved as part of the innate immune system as recognition molecules for pathogens and pathogen-infected cells. ${ }^{4}$

An interesting aspect of CD36 biology is that its expression on macrophages is increased when the cells are exposed to oxLDL. Among the changes that occur in the lipid components of LDL when it is oxidized is the formation of oxidized fatty acids such as 9- and 13-hydroxy octadecadienoic acid (HODE). These oxidized fatty acids are ligands for the nuclear hormone receptor peroxisome proliferator-activated receptor (PPAR) gamma, a transcription factor that regulates expression of many genes, including CD36. Thus, oxLDL promotes increased expression of CD36 and further cellular uptake of oxLDL. This feed-forward loop presumably accelerates foam cell formation in the arterial neointima. Furthermore, CD36 expression is upregulated at the transcriptional level by inflammatory cytokines such as granulocyte macrophage colony-stimulating factor (GM-CSF), macrophage colony-stimulating factor (M-CSF), and interleukin-4. Hyperglycemia increases CD36 expression through a nontranscriptional mechanism and may contribute to the proatherosclerotic state associated with diabetes.

\section{CD36 mediates atherogenesis}

The pathogenic role of oxLDL in atherosclerosis is largely dependent on CD36. Studies using macrophages from genetically altered mice developed in our laboratory that do not express CD36 demonstrated that absence of CD36 expression was associated with a lack of foam cell formation in vitro when cells were exposed to oxLDL. Wild-type mice, in contrast, showed foam cell formation after 12 to 24 hours. $^{5}$

To demonstrate in vivo relevance of these findings, we crossed CD36-null mice with proatherogenic apoE-null mice. When fed an atherogenic Western diet, apoE-null mice develop aortic atherosclerosis within several weeks, in a pattern and histology that closely resembles the human disease. In our experiment, the mice that lacked both CD36 and apoE had a dramatic decrease in the volume of atherosclerosis. ${ }^{5}$ Further studies showed that the proatherogenic role of CD36 was highly likely mediated by the CD36 on macrophages, since transplantation of bone marrow from CD36-null mice into apoE-null mice had the same effect on atherosclerosis as seen in the apoE/ CD36-double-null mice. ${ }^{6}$

Scavenger receptor-dependent formation and progression of atherosclerosis is supported by findings of an abundance of oxidized phospholipids that serve as binding partners for CD36 in the plaque region of blood vessels, along with an absence of oxidized phospholipids in the nonplaque region of blood. The enrichment of oxidized phospholipid in the plaque allows CD36 to penetrate the plaque, whereas removal of CD36 drastically decreases the progression of atherosclerosis.

\section{Platelet activation in the setting of hyperlipidemia}

In addition to the formation and progression of plaque, CD36 may be involved in the terminal phases of atherosclerosis (ie, the thrombosis that occurs on a plaque) as a result of abundant CD36 expression on platelets. CD36, in fact, was discovered as a platelet protein and named platelet glycoprotein IV, although for many years the function of CD36 on platelets was not known.

Recent studies by Podrez and colleagues, along with our group, revealed that oxLDL binds to the surface of platelets in a concentration-dependent manner, whereas normal LDL does not. The binding of oxLDL to platelets can be blocked almost completely by inhibiting CD36 with an antibody; binding did not occur with platelets obtained from CD36-deficient mice or people. ${ }^{7}$ Importantly, exposure to oxLDL caused platelets to be activated via a highly specific cell-signaling pathway; low concentrations of oxLDL, such as those found in plasma of individuals with even modest hyperlipidemia, made platelets more sensitive to low doses of "classic" platelet agonists such as collagen and adenosine diphosphate (ADP). ${ }^{8}$ These studies suggest that platelet CD36 could serve as a mechanistic link between inflammation, oxidant stress, and hyperlipidemia to create a prothrombotic state. 
It has been known for some time through the work of Eitzman and others that apoE-null mice fed a Western diet are "hypercoagulable"; ie, they have shortened thrombosis times. ${ }^{9}$ This observation led us to investigate the role of CD36 in the hyperlipidemia-associated prothrombotic state. In one experiment, tail-vein bleeding times were measured in apoE-null and apoE/CD36-double-null mice fed a high-fat diet. Whereas the apoE-null animals had markedly shortened bleeding times ( $\sim 2$ minutes), the double CD36/apoE-null animals were normal ( 6-8 minutes).

To examine a model more reflective of pathologic thrombus formation (eg, heart attack, stroke), we induced carotid artery injury in mice by topical application of ferric chloride. This method induces oxidant injury to the endothelium and causes platelet-dependent carotid occlusion. With this model, thrombosis can be monitored in "real time" with a Doppler flow probe and video microscope. As with tail-vein bleeding time, we found that time to carotid occlusion was much shorter in apoE-null mice fed a high-fat diet than in mice fed a normal chow diet or in wild-type mice; further, this prothrombotic state was rescued by genetic ablation of CD36 expression.

\section{Possible role in thrombus formation}

More recent experiments from our lab have shown that CD36-null mice fed a normal chow diet have a subtle defect in thrombus formation when arteries or veins are subjected to relatively mild injury. ${ }^{10}$ This finding implies a potential role for CD36 in "normal" platelet function and perhaps the existence of an endogenous ligand for CD36 that is unrelated to hyperlipidemia. Since we know that CD36-null mice and CD36-deficient people do not have a bleeding disorder and have normal bleeding times, it is possible that pharmacologic targeting of CD36 may provide a means to inhibit thrombosis without having a major impact on hemostasis.

The possibility that mice or humans can be protected from developing thrombi by blocking CD36 function is supported by initial data obtained from the carotid artery injury model in mice. In the laboratory, an antithrombotic state can be created by blocking the specific CD36-signaling pathway described below. Thrombocytopenic wild-type mice transfused with platelets from wild-type mice exhibit a dramatic increase in thrombosis time when the donor platelets are pretreated with a CD36-signaling inhibitor. ${ }^{8}$ This protective effect vanishes when the same experiment is performed in CD36-null mice.
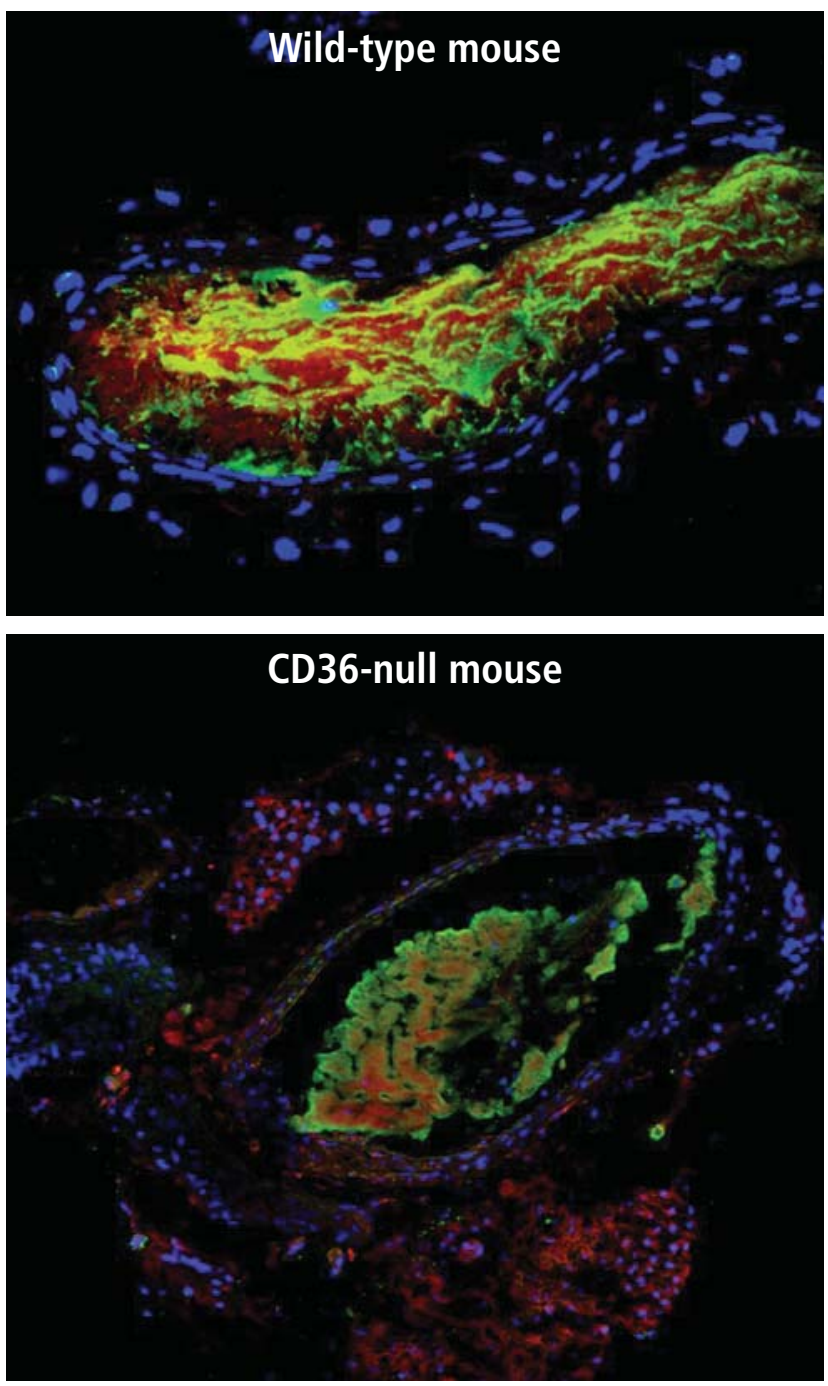

FIGURE 1. A mouse carotid artery (in cross section) injured with ferric chloride and then analyzed using immunofluorescent microscopy and an antibody to the endothelial cell-specific antigen CD105. The blue dots represent the nuclei in the vessel (stained with DAPI). In the top panel, a carotid thrombus in the lumen of a wildtype mouse is heavily enriched with CD105 (stained red), implying microparticle incorporation. In the CD36-null mouse (bottom panel), CD105 staining is decreased dramatically. The green staining represents an antibody to the platelet-specific antigen CD61. ${ }^{10}$

Reprinted from Ghosh A, Li W, Febbraio M, et al. Platelet CD36 mediates interactions with endothelial cell-derived microparticles and contributes to thrombosis in mice.

J Clin Invest 2008; 118:1934-1943. Copyright 2008 by American Society for Clinical Investigation. Reproduced with permission from American Society for Clinical Investigation.

\section{MICROPARTICLES: MAJOR LIGAND FOR CD36}

Based on the experiments described above, we hypothesized the existence of endogenous CD36 ligands involved in thrombosis and proposed that cellderived microparticles (MPs) were likely candidates. MPs are small (200-1,000 nm) phospholipid vesicles that "bud" off from cells as a result of stimulation or 
apoptosis. MPs can be derived from endothelial cells, leukocytes, cancer cells, and platelets; they contain selected membrane receptors as well as other proteins inherent to their parental cell (eg, MPs derived from a white cell contain tissue factor that can activate the coagulation cascade). MPs are known to circulate in patients with chronic inflammatory disorders, including acute coronary syndromes, lupus erythematosus, Wegener granulomatosis, and rheumatoid arthritis, and their number probably increases with aging.

Our hypothesis is based on the well-known observation that a major feature of MP generation is a loss of membrane asymmetry; that is, the PS normally expressed on the inner limit of the membrane instead is expressed on the surface. Previous studies from our lab and others had shown that PS and oxPS can be a ligand for CD36. ${ }^{11}$

To test our hypothesis we developed a rapid flow cytometry assay using an antibody to CD105, an antigen expressed only on endothelial cells, to detect an interaction between endothelial cell-derived MPs and platelets. This interaction is CD36-dependent in that it can be blocked with antibodies to CD36 and does not occur if platelets are taken from mice or humans who are CD36-deficient. ${ }^{10}$ MPs behaved like oxLDL in that platelets pretreated with MPs undergo a dramatic augmentation of aggregation in response to low doses of classic agonists. This augmentation of platelet activation does not occur in platelets from CD36-null donors or CD36-null mice. ${ }^{10}$

\section{Microparticle accumulation in thrombi}

To confirm a role for the platelet-MP interaction during thrombus formation, we examined CD105 antibody staining in thrombi induced in carotid arteries in our mouse models. We found that the endothelial cell-specific CD105 antigen accumulated in the thrombi formed in wild-type mice, but that staining was dramatically reduced in the thrombi formed in CD36-null animals (Figure 1). ${ }^{10}$

\section{Specific cytoplasmic signaling cascade}

When CD36 binds to its ligands (oxLDL or MPs), it transmits a signal to the cell. In macrophages this signal leads to oxLDL internalization and foam cell formation, while in platelets it contributes to platelet activation and aggregation. In a series of studies from our laboratory and others, it has been shown that these signals are relayed by a series of molecular interactions that involve specific tyrosine kinases from the Src family and serine/threonine kinases from the mitogen- activated protein (MAP) kinase family. ${ }^{12}$ The signal to the platelet is mediated by a MAP kinase called c-Jun N-terminal kinase (JNK). Carotid thrombi in wild-type mice stain for the presence of the activated, phosphorylated form of JNK, whereas phospho-JNK expression is decreased by $50 \%$ to $60 \%$ in carotid thrombi in CD36-null mice, ${ }^{12}$ similar to the decrease in MP mass in thrombi from CD36-null mice.

\section{CONCLUSION}

These experiments suggest that CD36 has both a proatherogenic and a prothrombotic role in the vascular system. Macrophage CD36 promotes foam cell formation and plaque formation. Platelet CD36 promotes thrombosis by signaling in response to oxLDL and by phospholipids present in cell-derived MP. Therefore, targeting CD36 or CD36-signaling pathways could be a strategy in the treatment of atheroinflammatory disorders and deserves exploration.

\section{REFERENCES}

1. Tontonoz P, Nagy L, Alvarez JG, Thomazy VA, Evans RM. PPAR-gamma promotes monocyte/macrophage differentiation and uptake of oxidized LDL. Cell 1998; 93:241-252.

2. Steinberg D. Lewis A. Conner Memorial Lecture. Oxidative modification of LDL and atherogenesis. Circulation 1997; 95:1062-1071.

3. Silverstein RL, Febbraio M. CD36 and atherosclerosis. Curr Opin Lipidol 2000; 11:483-491.

4. Krieger M. The other side of scavenger receptors: pattern recognition for host defense. Curr Opin Lipidol 1997; 8:275-280.

5. Febbraio M, Podrez EA, Smith JD, et al. Targeted disruption of the Class B scavenger receptor, CD36, protects against atherosclerotic lesion development in mice. J Clin Invest 2000; 105:1049-1056.

6. Febbraio M, Guy E, Silverstein RL. Stem cell transplantation reveals that absence of macrophage CD36 is protective against atherosclerosis. Arterioscler Thromb Vasc Biol 2004; 24:2333-2338.

7. Podrez EA, Byzova TV, Febbraio M, et al. Platelet CD36 links hyperlipidemia, oxidant stress and a prothrombotic phenotype. Nat Med 2007; 13:1086-1095.

8. Chen K, Febbraio M, Li W, Silverstein RL. A specific CD36dependent signaling pathway is required for platelet activation by oxidized LDL. Circ Res 2008; 102:1512-1519.

9. Eitzman DT, Westrick RJ, Xu Z, Tyson J, Ginsburg D. Hyperlipidemia promotes thrombosis after injury to atherosclerotic vessels in apolipoprotein E-deficient mice. Arterioscler Thromb Vasc Biol 2000; 20:1831-1834.

10. Ghosh A, Li W, Febbraio M, et al. Platelet CD36 mediates interactions with endothelial cell-derived microparticles and contributes to thrombosis in mice. J Clin Invest 2008; 118:1934-1943.

11. Greenberg ME, Sun M, Zhang R, Febbraio M, Silverstein RL, Hazen SL. Oxidized phosphatidylserine-CD36 interactions play an essential role in macrophage-dependent phagocytosis of apoptotic cells. J Exp Med 2006; 203:2613-2625

12. Rahaman SO, Lennon DJ, Febbraio M, Podrez EA, Hazen SL, Silverstein RL. A CD36-dependent signaling cascade is necessary for macrophage foam cell formation. Cell Metab 2006; 4:211-221.

Correspondence: Roy L. Silverstein, MD, Department of Cell Biology, Cleveland Clinic, 9500 Euclid Avenue, NC10, Cleveland, $\mathrm{OH}$ 44195; silverr2@ccf.org 